\section{e0434 THE EFFECTS OF PROTON PUMP INHIBITORS ON CLOPIDOGREL EFFICACY IN PATIENTS WITH ACS THROUGH PCI IN CHINA}

doi:10.1136/hrt.2010.208967.434

${ }^{1}$ Ren Yihong, ${ }^{1}$ Chen Yundai, ${ }^{1}$ Zhao Ming, ${ }^{2}$ Wang Chengbin, ${ }^{1}$ Chen Lian, ${ }^{1}$ Liu Hongbin, ${ }^{1}$ Wang Yu, ${ }^{1}$ Snu Zhijun, ${ }^{1}$ Chen Jinsong, ${ }^{1}$ Huang Tingting, ${ }^{1}$ Guo Yusong, ${ }^{1}$ Xie Yongjin. ${ }^{1}$ Pla General Hospital/cardiovascular Department; ${ }^{2}$ Pla General Hospital/clinical Laboratory

Background Almost all kinds of proton pump inhibitors (PPI), especially Omeprazole, have been reported to inhibit the antiplatelet activation of clopidogrel in the therapy of coronary artery disease and were associated with a higher risk of major cardiovascular events ranging from a $24.3 \%$ with lansoprazole to a $29.2 \%$ with pantoprazole in the increased risk. But recently, two clinical trials give us a paradoxical result from the above in nearly 13,800 patients with ACS. So, what the true influences of PPI on clopidogrel activation are still unclear. And especially, it has not been reported before in ACS group through PCI in Chinese patients. Objectives: To assess the effects of omeprazole on clopidogrel efficacy in patients with ACS through PCI in Chinese patients.

Methods In this randomised controlled trial, all patients $(n=186)$ with ACS and elective PCI who received aspirin (loading dose $300 \mathrm{mg}$ before PCI, followed by maintaining dose $100 \mathrm{mg}$ /day) and clopidogrel (loading dose $600 \mathrm{mg}$ before PCI, followed by maintaining dose $75 \mathrm{mg} /$ day) were randomised to receive omeprazole (20 mg/day) or placebo for 7 days. Residual platelet activity and platelet activation inhibition rate in $\mathrm{ADP}$ pathway were detected in the third day after PCI with modified thrombelastograpy-mapping (TEG-mapping) in ADP induced method.

Results Between the two groups with and without omeprazole, the mean platelet activation inhibition rate with ADP induced method is $63.52 \% \pm 23.11 \%$ vs $67.26 \pm 24.17(p=0.2895)$ detected with TEG, respectively. But when we divided the patients into 5 levels according to the clinical meaning of platelet activation inhibition rate, the frequency distribution in these 5 levels in the tow groups showed significant difference $(p=0.0062)$, especially the decrease of frequency in higher platelet activation inhibition rate with omeprazole. But without any changes can be seen in the distribution of highest or lowest inhibiting levels group.

Conclusion Without any significant effects of omeprazole on clopidogreal in total strength of inhibition rate to platelet can be observed in patients with ACS through PCI taking clopidogrel with $600 \mathrm{mg}$ loading dose and aspirin with $300 \mathrm{mg}$ loading dose. But omeprazole decrease the frequency distribution of higher platelet inhibition rate induced by clopidogrel significantly without increasing clopidogrel non-responds rate.

\section{e0435 THE DOUBLEFACED METABOLIC AND INFLAMMATORY EFFECTS OF STANDARD DRUG THERAPY IN PATIENTS AFTER DRUGELUTING STENT IMPLANTATION}

doi:10.1136/hrt.2010.208967.435

Liu Weimin, Wang Lijun, Wu Yue, Liu Xiaojun, Yuan Zuyi. First Affiliated Hospital of Medical College Xi'an Jiaotong University

Objective The inflammatory response and the long-term endothelium healing after drug-eluting stent (DES) placement has recently emerged as a major concern. We investigated the change of metabolic parameters and systematic inflammatory status of circulating mononuclear cells (MNC) in patients after coronary DES implantation.

Methods 27 patients with non-ST segmental elevation acute coronary syndrome that had undergone DES implantations were consecutively recruited and administrated with standard drug therapy for 12 weeks. The systematic inflammation on MNC was measured before DES implantations and after 12 weeks' medication. NF- $\mathrm{KB}$ binding activity in MNC and expression of its subunits p65 and $\mathrm{p} 50$, and depressed cytosolic I $\mathrm{KB}$ expression of MNC were detected to reveal the systematic inflammation after DES implantations.

Results Metabolic parameters total cholesterol, triglycerides, HDL, LDL improved significantly after 12 weeks' standard medication, but plasma concentrations of interleukin- 6 , tumour necrosis factor- $\alpha$, migration inhibitor factor, and matrix metalloproteinase- 9 increased compared with baseline $(p=0.012,0.035,0.062$ and 0.112 , respectively). The NF-kB DNA binding activity in MNC increased significantly compared with baseline $(p=0.015)$, concomitantly with decreased IKB- $\beta(p=0.052)$ and PPAR $-\gamma$ in MNC $(p=0.002)$. Although there were strong correlations within the change of metabolic parameters and within the change of proinflammatory factors, no significant correlations between them were observed.

Conclusions Standard drug therapy can improve metabolic parameters, but fail to restrain the deteriorated systemic inflammatory responses prolonged at least 3 month. These findings raise concern about the efficiency of the current standard therapy in the era of DES. However, longer term endpoint-based studies are necessary to further explore the relationship between the inflammatory factors and the clinical cardiovascular events.

\section{e0436 CLINICAL STUDY ON RELATIONSHIP BETWEEN THE PLASMA LTB4 LEVELS AND UNSTABLE PLAQUE IN ACUTE CORONARY SYNDROME}

doi:10.1136/hrt.2010.208967.436

Yang Lixia, Qi Feng, Guo Ruiwei, Wang Xianmei, Guo Chuanming. Department of Cardiology Kunmin General Hospital of Chinese Plakunming

Objective To explore the association between plasma Leukotriene B4 (LT-B4) and the severity of coronary artery lesions in coronary heart disease (CHD) patients.

Methods 400 patients were divided into CHD group $(n=310)$ and control group $(n=90)$ according to the Coronary Angiography (CAG). 310 patients in CHD group divided into Acute Coronary Syndrome (ACS) group $(\mathrm{n}=217)$ and Stable Angina Pectoris (SAP) group $(n=93)$ according to clinical information. The severity and extent of coronary lesions was analysed by CAG and typified by means of Gensini coronary score system.LT-B4 level in plasma of all patients were tested by Enzyme linked immunosorbent assay. And blood pressure, height, weight, body mass index $(\mathrm{BMI})=\mathrm{kg} / \mathrm{m} 2$ were measured at the same time. 85 cases of coronary heart disease patients were underwent 64 -slice spiral CT coronary artery imaging. Then, we evaluate the main plaque in Coronary artery.

Results HDL-C in CHD group was significantly lower than that in control group, but BMI, TC, TG, LDL-C, GLu and uric acid increased significantly compared with the control group. LT-B4 level in CHD patients increased significantly compared with the control group $(p<0.05)$.The concentrations of LT-B4, LDL- C and GLu in ACS group were increased significantly compared with SAP group, HDL$\mathrm{C}$ in ACS group was significantly lower than that in the SAP group $(p<0.05)$. As the the change of lesion type and the increasing of the severity of coronary artery disease, LT-B4 concentration increased gradually $(p<0.05)$. The plasma LT-B4 levels in soft plaque group and fibrous plaque group were much higher than that in calcified plaque group in CHD patients, and the difference was statistically significant $(p<0.05)$. Stepwise regression analysis showed that the concentration of LT-B4 was significantly correlated with Gensini score of coronary lesions. 